
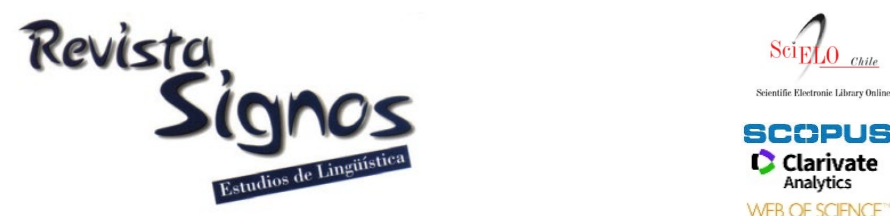

\title{
Desarrollo de la conciencia fonológica en el inicio del proceso de aprendizaje de la lectura
}

\author{
Development of the phonological awareness in the beginning of \\ the process of learning to read
}

\author{
Raúl Gutiérrez-Fresneda María Isabel De \\ UNIVERSIDAD DE ALICANTE \\ ESPAÑA \\ raul.gutierrez@ua.es
}

\author{
Rafael Alarcón Postigo \\ UNIVERSIDAD DE MÁLAGA \\ ESPAÑA \\ ralarcon@uma.es
}

Recibido: 04-VIII-2018 / Aceptado: 25-III-2020

DOI: $10.4067 /$ S0718-09342020000300664

\section{Resumen}

Las investigaciones de los últimos años han puesto de manifiesto que la conciencia fonológica constituye un predictor importante en la adquisición de la lectura y que además facilita su aprendizaje. Se sabe también que su desarrollo no eclosiona de manera innata, sino que se precisa de un entrenamiento explícito y progresivo de los diferentes niveles de los que se compone. La influencia del conocimiento fonológico y la lectura se han estudiado mayoritariamente cuando el aprendiz ya sabe leer, sin embargo, falta profundizar en estas relaciones en el inicio de este aprendizaje. El objetivo del presente estudio ha sido analizar las facetas que intervienen en el desarrollo de la conciencia fonológica cuando el niño emprende las primeras acciones decodificadoras con el propósito de identificar qué habilidades son las más adecuadas para fomentar el conocimiento fonológico en estos momentos. Se ha empleado un diseño descriptivo correlacional con la intención de analizar qué aspectos del conocimiento fonológico intervienen en mayor medida en el inicio del aprendizaje lector. En el estudio han participado 345 alumnos de diferentes centros públicos y concertados con edades comprendidas entre los 3 y los 4 años. Los resultados ponen de manifiesto que el desarrollo de la conciencia fonológica se incrementa cuando se inicia el proceso de aprendizaje de la lectura y que un factor que debe tenerse en consideración por su gran relevancia es el tipo de habilidades fonológicas a desarrollar ya que no todas presentan el mismo grado de complejidad en el periodo inicial del aprendizaje de la lectura.

Palabras Clave: Lectura, lenguaje escrito, alfabetización, conocimiento fonológico, enseñanza de la lectura. 


\begin{abstract}
Research in recent years has shown that phonological awareness is an important predictor in the acquisition of reading and also facilitates its learning. It is also known that its development does not hatch in an innate manner, but that it requires an explicit and progressive training of the different levels of which it is composed. The influence of phonological knowledge and reading have been studied mainly when the apprentice already knows how to read; however, it is necessary to deepen these relationships at the moment in which this learning begins. The purpose of this study has been to analyze the facets that most often intervene in the development of phonological awareness when the child performs the first decoding actions, as well as identify which skills contribute the most to phonological development in the first ages. A cross-sectional design has been used that has allowed us to clarify the factors of phonological knowledge that intervene in reading development to a greater extent. 345 students from different public and private schools with ages between 3 and 4 years participated in the study. The results show that the development of phonological awareness increases when the learning process of reading begins and that a factor that must be taken into consideration is the type of phonological skills to be developed since not all of them have the same degree of complexity in the initial period of learning to read.
\end{abstract}

Key Words: Reading, written language, literacy, phonological knowledge, teaching reading.

\title{
INTRODUCCIÓN
}

El aprendizaje de la lectura es una tarea de gran complejidad que requiere de varios años de instrucción y el dominio de una serie de habilidades para su adecuado aprendizaje, una de las habilidades más importantes es la conciencia fonológica, estando relacionada su adquisición tanto con el éxito en el proceso de alfabetización como con las dificultades de este aprendizaje (Suárez-Coalla, García de Castro \& Cuetos, 2013; Bowyer-Crane, Fricke, Schaefer, Lervag \& Hulme, 2017; González, López, Cuetos \& Vilar, 2017; Bar-Kochva \& Nevo, 2018). La conciencia fonológica está formada por diferentes componentes los cuales a su vez presentan distintos niveles de complejidad, en función de la unidad lingüística objeto de reflexión y manipulación, así como por los procesos que se realicen sobre ella (Treiman \& Zukowski, 1991; Bravo, 2004; Gutiérrez \& Díez, 2015).

Desde el punto de vista evolutivo, la conciencia fonológica se desarrolla principalmente desde los 3 hasta los 8 años de edad (Aguilar, Marchena, Navarro, Menacho \& Alcalde, 2011; Arancibia, Bizama \& Sáez, 2012) aunque no existe acuerdo en cómo se produce este desarrollo en cada uno de los momentos del aprendizaje del lenguaje escrito (Gutiérrez, 2018).

Durante los últimos años muchos han sido los estudios efectuados en torno a cómo el desarrollo fonológico afecta al aprendizaje lector (Calderón, Carrillo \& Rodríguez, 2006; Defior \& Serrano, 2011; Vicente-Yagüe, 2016; Rendón, García \& Navarro, 2019), estos se han llevado a cabo mayoritariamente sobre estudiantes que ya se habían iniciado en la lectura, sin embargo, son escasos los trabajos orientados en 
analizar el modo en el que la conciencia fonológica influye en el proceso de alfabetización en el momento en el que los niños comienzan este aprendizaje. Analizar cómo se va desarrollando esta habilidad en los momentos iniciales en los que los estudiantes toman contacto con el código escrito cuándo todavía no han sido instruidos en el conocimiento fonológico ha sido el objetivo de este estudio. Para ello se ha comparado a un grupo de aprendices que todavía no ha adquirido el código escrito junto con otros niños que ya se han iniciado en los primeros procesos decodificadores con el propósito de profundizar en cómo se va desarrollando la conciencia fonológica incluso antes de su ejercitación. Conocer esta situación es de gran relevancia ya que permitirá identificar qué tipo de acciones metafonológicas son las más adecuadas fomentar desde los momentos iniciales en los que se accede al aprendizaje del código escrito para facilitar el proceso de alfabetización.

\section{Marco teórico}

Una de las grandes dificultades que se encuentra al iniciarse en el aprendizaje de la lectura en un sistema alfabético como el castellano, es llegar a comprender la asociación que existe entre las letras (grafemas) y los sonidos del lenguaje hablado (fonemas) debido a que nuestro sistema de representación se basa en la segmentación de la cadena del habla, lo que requiere el desarrollo de habilidades fonológicas puesto que son estas las que facilitan la reflexión y la capacidad para manipular las unidades del lenguaje hablado (Arancibia et al., 2012; Gutiérrez \& Díez, 2018). La conciencia fonológica es la habilidad metalingüística que permite reflexionar sobre el lenguaje oral y segmentarlo en unidades menores (palabras, sílabas y fonemas), diferenciándose varios niveles de conciencia fonológica en función de la unidad de segmentación: conciencia léxica, cuando las unidades objeto de manipulación son las palabras; conciencia silábica, cuando son las sílabas sobre las que se realiza la acción y conciencia fonémica, cuando la unidad de segmentación son los fonemas. De estos niveles existe acuerdo en que la conciencia fonémica, es el aspecto que más relación presenta con el aprendizaje de la lectura debido a que cuanto mejor se identifiquen los elementos mínimos de las palabras más facilidad existirá para asociar los sonidos con sus correspondientes grafemas (Defior \& Serrano, 2011; Suárez-Coalla et al., 2013; Gutiérrez \& Díez, 2015; Gutiérrez, 2016; Jasińska \& Laura-Ann, 2017). De donde se deduce que la conciencia fonológica no es una habilidad única que emerge de una sola vez en un momento determinado, sino que constituye una habilidad conformada por varios componentes que se desarrollan en momentos distintos (Treiman \& Zukowski, 1996).

En la actualidad se sabe como consecuencia de los estudios de los últimos años que la conciencia fonológica es uno de los predictores más importantes en el aprendizaje de la lectura, así como en la explicación de sus dificultades (Bradley \& Bryant, 1983; Arnáiz, Castejón, Ruiz \& Guirao, 2002; Arancibia et al., 2011). Las evidencias que sustentan esta posición se basan en estudios longitudinales y trabajos 
de intervención que señalan cómo aprendices con dificultades en el proceso de aprendizaje de la lectura muestran avances a partir del entrenamiento en habilidades de conciencia fonológica (Bradley \& Bryant, 1985; Defior, 1990; Blachman, 1994; Domínguez, 1996; Meira, Cadime \& Leopoldina, 2019). Además, se ha comprobado que los niños con mejores habilidades para manipular sílabas o fonemas de las palabras aprenden a leer más rápido, independientemente del coeficiente intelectual, del vocabulario y del nivel socioeconómico (Gómez, Duarte, Merchán, Aguirre \& Pineda, 2007), mientras que por el contrario un déficit en esta habilidad genera diferencias significativas entre buenos y malos lectores (Márquez \& Osa, 2003).

Dado que la conciencia fonológica se compone de diferentes unidades lingüísticas (palabras, sílabas y fonemas), para determinar el nivel de competencia fonológica se debe atender a los distintos elementos del lenguaje ya que de esta depende la capacidad para manipular y tomar conciencia de las unidades del lenguaje oral (Gutiérrez \& Díez, 2018). Igualmente resulta relevante atender a los tipos de tareas fonológicas sobre las que se realiza la acción ya que no todas presentan el mismo nivel de complejidad, de hecho, se ha establecido una progresión de dificultad que comienza por aquellas situaciones que implican la identificación de diferencias ('tareas pasivas') hasta otras de mayor complejidad que requieren la manipulación directa sobre las unidades lingüísticas ('tareas activas') (Bravo, 2004).

Una vez conocida la importancia que presenta la conciencia fonológica en el aprendizaje de la lectura, una cuestión que debe analizarse es si la conciencia fonológica presenta un progreso evolutivo en las primeras edades y en caso afirmativo de qué modo se produce este desarrollo. Diferentes autores consideran que este aspecto es muy importante no solo por la relevancia que presenta sino porque actualmente no existe un consenso en cómo se desarrolla este proceso (Calderón et al., 2006). Unos consideran que la aparición de la conciencia fonológica tiene lugar en torno a la edad de 3 o 4 años (Aguilar, et al., 2011), otros afirman que se desarrolla entre el periodo comprendido entre los 4 y los 8 años y va desde la conciencia silábica hasta el manejo de las habilidades fonémicas una vez que se aprende a leer (Anthony $\&$ Francis, 2005). Arancibia et al. (2012) defienden que el periodo principal del desarrollo fonológico va desde los 4 años hasta el primer nivel de la escolaridad obligatoria. Defior y Serrano (2011) afirman que a los 4 años los niños ya pueden manipular las unidades silábicas, pero hasta el inicio de la escolaridad formal (6-7 años) no son capaces de reflexionar y manipular las unidades más pequeñas. Pérez y González (2004) indican que sobre los tres años los niños pueden reconocer dos palabras que riman, pero hasta los 8 años no adquieren la capacidad para eliminar sonidos dentro de las palabras.

Existe consenso en la literatura respecto a la importancia que presenta la conciencia fonológica en el aprendizaje de la lectura, sin embargo, no se encuentra acuerdo respecto al momento evolutivo en el que el niño se encuentra más capacitado 
para la adquisición de los diferentes niveles de los que se compone ni sobre cuándo debe iniciarse su desarrollo. Esclarecer este aspecto es un hecho relevante ya que ayudará a determinar el momento más idóneo para la enseñanza de la lectura en las primeras fases del proceso de alfabetización infantil, así como al diseño de programas que favorezcan el aprendizaje lector en las primeras edades.

La importancia de la conciencia fonológica en la adquisición de la lectura está ampliamente documentada cuando el aprendiz ya sabe leer (Thomson \& Hogan, 2009; Defior \& Serrano, 2011; González et al., 2017; Gutiérrez, 2018), sin embargo, apenas hay trabajos que analicen la influencia de las habilidades que favorecen el desarrollo del conocimiento fonológico en el momento en el que el niño realiza las primeras acciones decodificadoras. El objetivo de este estudio fue analizar las facetas que intervienen en el desarrollo de la conciencia fonológica cuando el niño se inicia en el aprendizaje de la lectura con el propósito de identificar qué habilidades son las más adecuadas para fomentar el conocimiento fonológico. Para ello, se evalúa el desarrollo de los distintos niveles de conciencia fonológica (léxico, silábico y fonémico) a través de distintas acciones metafonológicas (identificar, añadir, omitir) en dos muestras de alumnos de 3 y 4 años, que se están iniciando en el proceso de alfabetización y se diferencian por su nivel de aprendizaje de la lectura.

En este sentido y con la intención de poder ampliar el conocimiento sobre las facetas que intervienen en el desarrollo de la conciencia fonológica cuando el niño se inicia en el aprendizaje de la lectura se plantean las siguientes cuestiones de investigación: ¿qué tipo de tareas son las más adecuadas para iniciar el desarrollo de las habilidades fonológicas?, ¿es relevante la posición del elemento lingüístico sobre el que se realiza la acción cognitiva en las tareas fonológicas? Dar respuesta a estos interrogantes es un aspecto relevante para el diseño de programas educativos eficaces en las primeras edades cuando se inicia el proceso de alfabetización.

\section{Marco metodológico}

\subsection{Participantes}

En el estudio participaron 345 alumnos con edades comprendidas entre los 3 y los 4 años $(M=3.6 ; D T=0.4)$, de los cuales el $49,2 \%$ eran niños y el $50,8 \%$ niñas, todos ellos pertenecientes a diferentes centros públicos y concertados de nivel sociocultural medio. Se distribuyeron en dos grupos, el grupo 1 estaba compuesto por 175 alumnos (el $48,3 \%$ eran niños y el $51,7 \%$ niñas) que no habían recibido instrucción en conciencia fonológica, aunque sí que se les había enseñado las vocales y algunas consonantes, pero no tenían adquirido este aprendizaje ya que no eran capaces de identificar correctamente su correspondencia grafema-fonema. El grupo 2 estaba formado por 170 estudiantes (el 49,5\% eran niños y el 50,5\% niñas) los cuales tampoco habían recibido instrucción en las habilidades fonológicas, pero sí que conocían las vocales y alguna consonante del código lingüístico del castellano y eran 
capaces de efectuar sencillas acciones decodificadoras a nivel silábico. Para el desarrollo del presente estudio se utilizó un método de muestreo probabilístico aleatorio para tener mayor representatividad de los resultados y poder generalizar a toda la población a la cual representa la muestra. El análisis de la potencia estadística permite la selección de un tamaño muestral que garantice el éxito de la investigación (Davey \& Savla, 2010), presentando la muestra en este estudio un valor de 0.914, con un error alfa de .05 .

\subsection{Instrumentos}

Se administraron a todos los participantes de la muestra de manera individual en un aula próxima a la clase ordinaria, las pruebas que a continuación se reseñan con el propósito de conocer el grado de dominio tanto de la conciencia fonológica como de los procesos decodificadores que se ponen en práctica en los momentos iniciales del aprendizaje de la lectura.

- Prueba de Segmentación lingüistica (PSL) (Jiménez \& Ortiz, 2000) en la que el niño debe identificar el número de palabras que tienen una serie de oraciones presentadas a nivel oral. La puntuación se obtiene asignando un punto a cada respuesta correcta. Con los datos del presente estudio a través del índice de fiabilidad compuesta $(\mathrm{FC}=.87)$ y la varianza media extractada (VME = $61,43 \%$ ) se comprueba que la prueba es fiable.

- Prueba para la evaluación del conocimiento léxico, se ha diseñado ad boc y se compone de dos partes, en la primera se valora la adicción léxica mediante la capacidad para construir oraciones verbalmente a partir de una palabra dada, mientras que en la segunda se valora la omisión léxica a través de la capacidad para suprimir palabras a una serie de oraciones presentadas oralmente. Cada una de estas tareas se compone de ocho ítems, concediéndose un punto por cada respuesta correcta. Se calculó la fiabilidad compuesta (FC) y la varianza media extractada (VME) con los datos del presente estudio. Los resultados mostraron que la fiabilidad fue elevada $(\mathrm{FC}=.92)$, y la varianza media extractada fue superior a $.50(\mathrm{VME}=57,62 \%)$ lo que implica que un alto porcentaje de la varianza es explicada por el constructo.

- Prueba para la Evaluación del Conocimiento Fonológico (PECO) (Ramos \& Cuadrado, 2006). Este test evalúa los niveles de conocimiento fonológico (silábico y fonémico), cada uno de los cuales se compone de tres tareas distintas: identificación, adición y omisión. Esta prueba incluye tres subtests con sílabas y fonemas (actividades de identificación, adición y omisión), con un total de 30 ítems (15 de sílabas y 15 de fonemas). La puntuación máxima que puede obtenerse es 30, un punto por cada respuesta correcta y cero por cada error. La confiabilidad, medida a través del coeficiente alfa de Cronbach es de .80. Con los datos del presente estudio a través del índice de fiabilidad compuesta (FC = 
.87) y la varianza media extractada $(\mathrm{VME}=63,58 \%)$ es posible comprobar que la prueba es fiable.

- Batería de inicio a la lectura (BIL). (Sellés, Martínez, Vidal-Abarca \& Gilabert, 2008) con la finalidad de valorar el grado de identificación del conocimiento alfabético para lo cual se empleó la subprueba: conocimiento del nombre de las letras. Esta prueba presenta un coeficiente de fiabilidad de Cronbach de 0.78. Además, se elaboró una prueba ad hoc formada por diez unidades silábicas con las letras trabajadas, cinco de estructura consonante-vocal (mi, le, po, mu, pa) y otras cinco de estructura consonante-vocal-consonante (pol, mal, pel, pil, mul). La puntuación se obtiene asignando un punto a cada respuesta correcta. Con los datos del presente estudio a través del índice de fiabilidad compuesta ( $\mathrm{FC}=$ .91) y la varianza media extractada $(\mathrm{VME}=62,41 \%)$ se comprueba que la prueba es fiable.

\subsection{Procedimiento}

El diseño del estudio utilizado para esta investigación es de tipo descriptivo y correlacional (Coolican, 1994). Es un estudio descriptivo porque se propone medir el constructo de conciencia fonológica, entendido como un conjunto de distintas unidades léxicas y subléxicas (palabras, sílabas y fonemas) y correlacional porque se intentan establecer relaciones entre el rendimiento de distintos grupos de alumnos en una serie de pruebas que evalúan la conciencia fonológica con el propósito de analizar las variables objeto de estudio: conciencia léxica, conciencia silábica y conciencia fonémica, al ser estas habilidades las que han demostrado su positiva influencia en el proceso de aprendizaje de la lectura.

La aplicación de las pruebas se llevó a cabo por profesionales de la educación (docentes previamente entrenados) lo que facilitó la homogeneidad en la recogida de los datos. La valoración de los alumnos se realizó de forma individual en espacios próximos al aula ordinaria en el tercer trimestre del curso dentro del horario escolar. Ninguno de los alumnos participantes en el estudio había sido previamente entrenado explícitamente en ninguna de las habilidades fonológicas (conciencia léxica, silábica o fonémica) aunque sí que habían realizado algunas actividades de estimulación de expresividad oral y se les había iniciado en la enseñanza del lenguaje escrito, en concreto en los procesos de correspondencia de las vocales y de algunas consonantes. El estudio respetó los valores éticos requeridos en la investigación con seres humanos (consentimiento informado, protección de datos personales, no discriminación, gratuidad y tener la posibilidad de abandonar el programa en cualquiera de sus fases).

\section{Resultados}

Para el análisis de los resultados se tuvo en consideración el grado de conocimiento alfabético, en concreto de las cinco vocales y de al menos tres consonantes, 
atendiéndose para la distribución de los alumnos al nivel de dominio que presentaban respecto al proceso decodificador de las unidades silábicas de estructura: consonantevocal y consonante-vocal-consonante.

A partir del grado de aprendizaje de la lectura de estas unidades mínimas se distribuyeron a los estudiantes en dos grupos. En el grupo 1 se encontraban los alumnos que no podían identificar correctamente la correspondencia grafema-fonema de las cinco vocales y algunas consonantes y por tanto no eran capaces de realizar acciones decodificadoras a nivel silábico. El grupo 2 estaba formado por los estudiantes que conocían las vocales y al menos tres consonantes del código lingüístico del castellano y eran capaces de efectuar sencillas acciones decodificadoras a nivel silábico.

Para el análisis de los niveles de dificultad de las habilidades que contribuyen al desarrollo de la conciencia fonológica se valoró el grado de desempeño alcanzado respecto a tres tipos de acciones cognitivas: identificación, adición y omisión mediante la manipulación de las tres unidades principales de manipulación y segmentación del lenguaje oral: léxicas, silábicas y fonémicas. Para favorecer su estudio se ha seguido el procedimiento de análisis convencional de ítems (Martínez-Arias, 1995) en el que se establecen los valores que limitan las categorías, siendo: muy fáciles, los valores mayores de 0.74; fáciles, los ubicados entre 0.73 y 0.55 ; de dificultad media los situados entre 0.54 y 0.45 ; difíciles los que se encontraban entre 0.44 y 0.25 y muy difíciles los menores de 0.24 .

Observando los resultados globales (Tabla 1), se comprueba que la conciencia silábica resulta más fácil que la conciencia léxica y fonémica en todos los estudiantes, siendo esta última la que mayor grado de dificultades presenta en ambos grupos. De igual modo, destaca el hecho de que para el grupo 2 todas las tareas presentan menor grado de complejidad que para el alumnado que todavía no se ha iniciado en el proceso decodificador (grupo 1). Si consideramos de manera general los tres tipos de tareas, la identificación es la acción cognitiva más sencilla seguida de la adición, siendo la omisión la tarea que conlleva mayor complejidad para todos los alumnos.

En cuanto a la unidad lingüística sobre la que se efectúa la manipulación cognitiva, para el grupo 1 (alumnado que todavía no domina los procesos decodificadores) las tareas con palabras y sílabas entran en la categoría de difíciles, mientras que los fonemas se encuadran dentro del nivel de dificultad alta, mientras que para el grupo 2 (alumnos que se han iniciado en el proceso decodificador) todas las tareas se engloban dentro de la categoría de difíciles, pero ninguna como muy difíciles.

Respecto al tipo de tarea, para el grupo 1 las acciones cognitivas de identificación y adición entran en la categoría de difíciles y las de omisión de dificultad alta, por otra parte, para el grupo 2 las acciones de identificación presentan un grado de dificultad 
media, mientras que la adición y la omisión forman parte de la categoría de difíciles, pero no resultan muy difíciles como sucedía en el grupo 1.

Tabla 1. Índices de dificultad totales en las tareas de identificación, adición y omisión con palabras, sílabas y fonemas.

\begin{tabular}{|l|c|c|}
\hline & Grupo 1 & Grupo 2 \\
\hline Total tareas con palabras & 0.39 & 0.44 \\
\hline Total tareas con sílabas & 0.40 & 0.45 \\
\hline Total tareas con fonemas & 0.21 & 0.26 \\
\hline Total tareas de identificación con palabras, sílabas y fonemas & 0.41 & 0.47 \\
\hline Total tareas de adición con palabras, sílabas y fonemas. & 0.35 & 0.40 \\
\hline Total tareas de omisión con palabras, sílabas y fonemas. & 0.22 & 0.26 \\
\hline
\end{tabular}

En la Tabla 2 se presentan detallados todos los índices de dificultad de manera pormenorizada, pudiéndose observar que tanto las características de las acciones cognitivas a realizar en cada una de las tareas como la unidad lingüística sobre la que se realiza la manipulación fonológica constituyen aspectos relevantes en el desarrollo de la conciencia fonológica. Respecto al tipo de tarea se observa que las acciones de identificación son las que les resultan más sencillas a los aprendices seguida de las tareas de adición, mientras que la omisión es la acción fonológica de mayor complejidad. Además, en todos los casos la posición del elemento sobre el que se lleva a cabo la manipulación fonológica presenta una especial relevancia. En el caso de la identificación, es la posición inicial la que resulta de menor complejidad, seguidas de la final y medial respectivamente, tanto en el caso de las sílabas como de los fonemas. En la adición, por el contrario, es la posición final la que presenta menor complejidad seguida de la inicial tanto en el caso de las sílabas como de los fonemas. Mientras que son bastante más complejas las acciones que implican añadir elementos tanto de carácter silábico como fonémico en medio de la palabra. Esta misma situación se produce en el caso de la omisión, resulta más fácil suprimir elementos al final de la palabra que al inicio y es mucho más difícil llevar a cabo estas acciones cuando las demandas de la tarea se encuentran en posición medial.

Respecto a la conciencia léxica, en todos los tipos de tareas se mantiene el mismo patrón, cuanto mayor es el número de elementos léxicos más compleja es la tarea.

Destaca el hecho de que en todas las tareas es el grupo que se ha iniciado en el proceso lector (grupo 2) el que logra un mayor dominio en el desarrollo de la conciencia fonológica en comparación con los alumnos que todavía no han adquirido el proceso decodificador.

Respecto al grado de dificultad de las diferentes tareas, los niños a la edad de 3 y 4 años se encuentran ya preparados para realizar distintas acciones metafonológicas, aunque no todas, ya que algunas presentan en estos momentos un alto grado de dificultad. En cuanto al desarrollo fonológico a través de las acciones de 
identificación, la conciencia léxica, para el grupo 1 es una tarea muy adecuada al no tener una elevada dificultad cuando intervienen dos palabras y tres palabras, al igual sucede en el caso del grupo 2 para quienes son muy fáciles las tareas de identificación con dos palabras y presentan una dificultad media las que implican el manejo de una unidad léxica más. En el caso de las oraciones de cuatro palabras, para ambos grupos este tipo de tareas presentan una complejidad muy alta, por lo que no sería recomendable su incorporación en estos momentos. El desarrollo de la conciencia silábica mediante las tareas de identificación resulta de gran facilidad en posición inicial para los estudiantes de ambos grupos, dificultad media en posición final y de gran dificultad en posición medial especialmente para los aprendices que todavía no realizan asociaciones de correspondencia grafema-fonema. La conciencia fonémica a través de las acciones metafonológicas de identificación presenta mayor complejidad que las tareas anteriores, aunque tanto la identificación inicial como final no presentan un grado de complejidad excesivamente elevado, lo que sí que sucede cuando la acción implica una manipulación a nivel medial.

El desarrollo fonológico a través de las tareas de adición presenta niveles diferentes en función del grado de dominio lector, en el caso de las oraciones de dos palabras son acciones de relativa facilidad, pero su complejidad aumenta a medida que se incrementan las unidades léxicas, con cuatro palabras el grado de dificultad es elevado.

El patrón encontrado indica que las acciones de adición resultan más fáciles en posición final que al inicio de las palabras tanto en el caso de las sílabas como de los fonemas, mientras que manejar estas unidades lingüísticas en medio de la palabra es de elevada complejidad tanto para el grupo de alumnos que no se ha iniciado en el proceso decodificador como para los que ya realizan acciones de lectura.

Por último, el desarrollo de la conciencia fonológica con oraciones de dos palabras puede ser ejercitado en estos momentos ya que presenta un grado de dificultad media, mientras que las oraciones de tres y cuatro palabras presentan unos parámetros de dificultad elevada para los estudiantes de los dos grupos. En el caso de la omisión silábica y fonémica el patrón encontrado es similar a las tareas anteriores, omitir sílabas y fonemas en posición final resulta más sencillo que al inicio de la palabra, aunque existe una diferencia notable en cuanto a la unidad lingüística sobre la que se realiza la acción. En el caso de las sílabas la dificultad para suprimir estas unidades en posición final es media y medio alta cuando la acción metafonológica se realiza en el inicio de las palabras, presentando una gran complejidad cuando la unidad a manipular se encuentra en posición medial. En el caso de los fonemas todas las acciones de omisión con independencia de la posición del elemento sobre el que se realice la acción manipulativa presentan un grado de gran dificultad en este momento del desarrollo para todos los estudiantes, se hayan iniciado o no en el proceso lector. 
Tabla 2. Índices de dificultad en cada una de las tareas de identificación, adición y omisión de palabras, sílabas y fonemas en los dos grupos de participantes.

\begin{tabular}{|c|c|c|c|c|c|c|c|}
\hline & \multicolumn{2}{|c|}{ Tareas de Identificación } & \multicolumn{2}{|c|}{ Tareas de Adición } & \multicolumn{2}{|c|}{ Tareas de Omisión } \\
\hline & & Grupo 1 & Grupo 2 & Grupo 1 & Grupo 2 & Grupo 1 & Grupo 2 \\
\hline \multirow{4}{*}{ Palabras } & 2 palabras & 0.78 & 0.85 & 0.64 & 0.72 & 0.45 & 0.55 \\
\hline & 3 palabras & 0.48 & 0.52 & 0.37 & 0.42 & 0.37 & 0.41 \\
\hline & 4 palabras & 0.07 & 0.11 & 0.26 & 0.28 & 0.12 & 0.15 \\
\hline & Media & 0.44 & 0.49 & 0.42 & 0.47 & 0.31 & 0.37 \\
\hline \multirow{4}{*}{ Sílabas } & 2 palabras & 0.91 & 0.95 & 0.43 & 0.51 & 0.37 & 0.42 \\
\hline & 3 palabras & 0.44 & 0.54 & 0.52 & 0.59 & 0.47 & 0.54 \\
\hline & 4 palabras & 0.22 & 0.26 & 0.17 & 0.19 & 0.06 & 0.08 \\
\hline & Media & 0.52 & 0.58 & 0.37 & 0.43 & 0.30 & 0.34 \\
\hline \multirow{4}{*}{ Fonemas } & 2 palabras & 0.50 & 0.63 & 0.34 & 0.39 & 0.07 & 0.08 \\
\hline & 3 palabras & 0.41 & 0.45 & 0.42 & 0.53 & 0.09 & 0.11 \\
\hline & 4 palabras & 0.07 & 0.12 & 0.02 & 0.04 & 0.02 & 0.03 \\
\hline & Media & 0.32 & 0.40 & 0.26 & 0.32 & 0.06 & 0.07 \\
\hline
\end{tabular}

Nota: El grado de dificultad de las tareas viene determinado por el procedimiento de análisis convencional de ítems (Martínez, 1995) que se corresponde con los siguientes valores:

Muy fáciles: $>0.74$

. Fáciles: $0.73-0.55$

. Dificultad media: $0.54-0.45$

. Difíciles: $0.44-0.25$

. Muy difíciles: $<0.24$

\section{Discusión}

El objetivo de este estudio fue analizar las facetas que en mayor medida intervienen en el conocimiento fonológico cuando se llevan a cabo las primeras acciones decodificadoras con la finalidad de identificar qué tareas metafonológicas son las más adecuadas para fomentar el conocimiento fonológico en el inicio del proceso de alfabetización.

Los resultados de este trabajo evidencian que la conciencia fonológica no es constructo único que surge en un momento determinado, sino que constituye un aprendizaje en el que están implicados distintos niveles y etapas que se desarrollan de manera evolutiva. En este sentido coincidimos con distintos autores (Diuk, Borzone \& Ledesma, 2010; Gutiérrez, 2018a) al señalar que la conciencia fonológica está formada por distintas habilidades, de diferente nivel de complejidad y que emergen progresivamente durante el desarrollo, lo que implica la necesidad de analizar con precisión los distintos niveles de dificultad de las operaciones cognitivas que se realizan para el desarrollo fonológico en función, por un lado, del tipo de tarea que se lleve a cabo, y por otro, del tipo de unidad lingüística sobre la que se realiza la acción.

Respecto a las tareas metafonológicas, en el presente estudio se han analizado tres tipos: identificación, adición y omisión y respecto a la unidad lingüística sobre la que se realiza la acción manipulativa se han considerado las tres principales, la palabra, la sílaba y el fonema. Se ha constatado a nivel general que el manejo de la sílaba resulta más fácil para el aprendiz que la manipulación de la palabra y el fonema y que la toma 
de conciencia de los fonemas de las palabras es la acción metafonológica más compleja, lo que coincide con los datos encontrados por otros autores (Defior \& Serrano, 2011; Gutiérrez, 2018b). Respecto al tipo de tarea empleada para el desarrollo fonológico es la identificación la acción cognitiva que resulta más sencilla a los estudiantes que se están iniciando en el proceso lector seguida de la adición y la omisión respectivamente. Estos aportes se hayan en consonancia con las afirmaciones de otros estudios previos que señalan que para aprender a leer en las diferentes lenguas no todas las unidades subléxicas (silabas, unidades intrasilábicas y fonemas) tienen la misma importancia durante el proceso de aprendizaje de la lectura (Ziegler \& Goswami, 2005).

De igual modo, es relevante destacar que no solo la tarea juega un papel determinante en el desarrollo fonológico, sino que la posición del elemento lingüístico sobre el que se realiza la acción cognitiva también resulta significativa. En este sentido, en el caso de las situaciones de identificación, es la posición inicial por la que se debería iniciar el entrenamiento al principio de la alfabetización ya que esta es la que resulta menos compleja a los estudiantes seguida de la posición final y medial respectivamente. En las operaciones cognitivas de adición y omisión con sílabas y fonemas son las acciones que implican la manipulación cognitiva de las unidades lingüísticas en posición final las tareas más sencillas y por tanto por las que se aconseja iniciar el desarrollo de la conciencia fonológica. Posteriormente y a partir del dominio de este tipo de tareas, es la posición inicial de la palabra la unidad lingüística por la que se debería continuar, dejando para más adelante las acciones manipulativas que se llevan a cabo en medio de la palabra por su alto nivel de dificultad. Estos datos se encuentran en consonancia con los encontrados en otros estudios realizados con alumnos mayores que ya se habían iniciado en el proceso lector en los que también se señala que la omisión medial tanto silábica como fonémica constituyen tareas de alta complejidad en los momentos iniciales del aprendizaje de la lectura (Aguilar, et al., 2011). En inglés, una lengua que presenta un gran número de irregularidades, aprender patrones como la rima resulta ventajoso para el lector inicial (Fumagalli, Barreyro, Borzone \& Jaichenco, 2014). En investigaciones realizadas en lengua inglesa se observa que los niños reconocen mejor los fonemas en posición inicial que los fonemas en posición final (Treiman \& Zukowski, 1991).

Otro aspecto relevante, ha sido analizar el desarrollo fonológico en función del grado de adquisición del código escrito de los estudiantes ya que no todos se encontraban en el mismo nivel de desarrollo lector. Los alumnos que todavía no eran capaces de realizar las primeras acciones decodificadoras han obtenido resultados inferiores a los de los compañeros que ya se habían iniciado en los procesos de correspondencia grafema-fonema. Este aspecto es relevante porque, aunque está bien establecida la vinculación entre el desarrollo fonológico y lectura cuando ya se sabe leer, existe en la actualidad una controversia sobre la dirección de esta relación, es 
decir, no se sabe si es la conciencia fonológica la que contribuye al aprendizaje de la lectura o a la inversa. Uno de los postulados que se plantea es que al igual que el conocimiento fonológico es una habilidad que favorece la adquisición de la lectura, este aprendizaje puede facilitar el desarrollo de la conciencia fonológica (Castles \& Coltheart, 2004), es decir, existirían distintas vías para el desarrollo del conocimiento fonológico, una sería aprender a leer y la otra la ejercitación de las habilidades fonológicas. Los datos de este estudio permiten avanzar en este postulado ya que cuando el aprendiz se inicia en el proceso lector en los niveles más elementales ha desarrollado en mayor grado las habilidades que favorecen el conocimiento fonológico, es decir, el aprendizaje inicial de la lectura contribuye desde los primeros momentos a un mayor desarrollo de la conciencia fonológica. Es igualmente destacable el hecho de que junto a las aportaciones que se vienen realizando últimamente sobre la influencia de la conciencia fonológica en la adquisición del código escrito, no es solo el desarrollo fonológico el que facilita el aprendizaje de la lectura, sino que el proceso decodificador también favorece la mejora de las habilidades fonológicas y lo que es más importante y se ha puesto de manifiesto en este trabajo, este hecho se produce desde los primeros contactos con el código escrito. Este hecho es relevante ya que dada la vinculación existente entre la conciencia fonológica y el aprendizaje de la lectura (Bradley \& Bryant, 1991; Jiménez \& Ortiz, 2000; Gutiérrez \& Díez, 2015) cuanto antes se inicie el acceso al aprendizaje del código escrito más se desarrollarán las habilidades favorecedoras de la conciencia fonológica que tan importantes son para el progreso por los diferentes niveles del aprendizaje lector, especialmente en sistemas ortográficos transparentes como el castellano, en los que existe una clara correspondencia entre los elementos sonoros (fonemas) y sus correspondientes grafemas.

\section{CONCLUSIONES}

El apoyo empírico que la conciencia fonológica presenta en el aprendizaje del lenguaje escrito refleja que los niños que presentan carencias al resolver tareas que requieren de esta habilidad al comienzo de la instrucción lectora obtienen menos éxito en este aprendizaje que aquellos que puntúan más alto. Este hecho se ha puesto de manifiesto tanto con estudiantes que ya sabían leer como con otros a los que se les ha instruido mediante programas orientados al desarrollo de la conciencia fonológica (Aguilar, et al., 2011; González et al., 2017). Pero además en este trabajo se ha podido conocer que el desarrollo fonológico tiene un papel relevante incluso antes del entrenamiento en esta habilidad lingüística desde los primeros contactos con el código escrito. De donde se deduce que la puesta en práctica de propuestas didácticas que combinen el desarrollo de las habilidades fonológicas junto al aprendizaje del conocimiento alfabético desde el inicio de este aprendizaje favorecerá en mayor medida la toma de conciencia de los segmentos del lenguaje hablado y a hacer explícita 
la conexión entre los sonidos del lenguaje oral y los símbolos gráficos que los representan.

Estos aportes son especialmente significativos ya que permiten contribuir a solventar una de las carencias que actualmente existen en el ámbito educativo y es que a pesar de las evidencias que presenta la conciencia fonológica en el aprendizaje de la lectura no se observa en las aulas de los primeros niveles escolares una puesta en práctica de intervenciones pedagógicas dirigidas a su desarrollo. Uno de los motivos puede ser el hecho de que, aunque se sabe que los niños están capacitados a partir de los tres años para iniciarse en el análisis de la estructura sonora del habla y se conocen los beneficios que presenta el conocimiento fonológico en los primeros momentos del aprendizaje lector no se han realizado estudios en los que se haya concretado de manera específica qué tipo de actividades son las más adecuadas implementar en cada periodo evolutivo del niño. Un hecho que puede ayudar a este logro es considerar las aportaciones reseñadas en este estudio en el que se indican con detalle cuales son las tareas más aconsejables para el desarrollo fonológico desde el instante en el que los escolares se inician en el proceso de alfabetización, qué unidades lingüísticas deben tenerse en consideración para tal fin, en qué secuencia deben abordarse para favorecer su aprendizaje y qué tipo de propuestas no se recomiendan implementar a determinadas edades por la elevada dificultad que presentan en estas primeras fases del aprendizaje, lo cual resulta fundamental para el diseño de programas eficaces que favorezcan el aprendizaje de la lectura en las primeras edades.

Ahora bien, es necesario que los aportes que se han ofrecido en este estudio tengan continuidad en los cursos posteriores ya que de este modo se podrá concretar con mayor precisión el desarrollo evolutivo de la conciencia fonológica durante las primeras etapas del aprendizaje de la lectura.

En resumen, este estudio contribuye a la facilitación de los procesos de aprendizaje de la lectura en las primeras edades mediante el diseño y la realización de actividades educativas que incidan en las habilidades que se han identificado como relevantes. Situación que es de gran interés habida cuenta de las repercusiones negativas que los problemas fonológicos y las dificultades de lectura presentan en el aprendizaje escolar. Una limitación de este trabajo es que no se atendió a determinadas variables, como son las prácticas lectoras realizadas en el ámbito familiar, la motivación e interés familiar hacia el aprendizaje lector, el desarrollo de los distintos componentes de desarrollo del lenguaje oral, que de igual modo pueden resultar determinantes en el proceso de aprendizaje de la lectura.

\section{REFERENCIAS BIBLIOGRÁFICAS}

Aguilar, M., Marchena, E., Navarro, J. I., Menacho, I. \& Alcalde, C. (2011). Niveles de dificultad de la conciencia fonológica y aprendizaje lector. Revista de Logopedia, Foniatría y Audiología, 31(2), 96-105. 
Anthony, J. \& Francis, D. (2005). Development of phonological awareness. Current Directions in Psychological Science, 14(5), 255-259.

Arancibia, B., Bizama, M. \& Sáez, K. (2012). Aplicación de un programa de estimulación de la conciencia fonológica en preescolares de nivel transición 2 y alumnos de primer año básico pertenecientes a escuelas vulnerables de la Provincia de Concepción, Chile. Revista Signos. Estudios de Lingüistica, 45(80), 236-256.

Arnáiz, P., Castejón, J. L., Ruiz, M. S. \& Guirao, J. M. (2002). Desarrollo de un programa de habilidades fonológicas y su implicación en el acceso inicial a la lecto-escritura en alumnos de segundo ciclo de educación infantil. Educación, desarrollo y diversidad, 5, 29-51.

Bar-Kochva, I. \& Nevo, E. (2018). The relations of early phonological awareness, rapid-naming and speed of processing with the development of spelling and reading: a longitudinal examination. Journal of Research in Reading, 42(1), 97-122. doi: $\underline{10.1111 / 1467-9817.12242}$

Blachman, B. (1994). What we have learned from longitudinal studies of phonological processing and reading, and some unanswered questions: A response to Torgesen, Wagner and Rashotte. Journal of Learning Disabilities, 77, 787-791.

Bowyer-Crane, C. A., Fricke, S., Schaefer, B., Lervag, A. \& Hulme, C. (2017). Early literacy and comprehension skills in children learning English as an additional language and monolingual children with language weaknesses. Reading and Writing, 30(4), 771-790. doi: https://doi.org/10.1007/s11145-016-9699-8

Bradley, L. \& Bryant, P. (1983). Categorizing sounds and learning to read: A causal connection. Nature, 301, 419-421.

Bradley, L. \& Bryant, P. (1985). Rhyme and reason in reading and spelling. AM Arbor: The University of Michigan Press.

Bradley, L. \& Bryant, P. E. (1991). Phonological skills before and after learning to read. En S. A. Brady \& D. P. Shankweiler (Eds.), Phonological processes in literacy (pp. 37-45). Hilldale: Erlbaum.

Bravo, L. (2004). La conciencia fonológica como un posible zona de desarrollo próximo para el aprendizaje de la lectura inicial. Revista Latinoamericana de Psicología, 36, 21-32.

Calderón, G., Carrillo, M. \& Rodríguez, M. (2006). La conciencia fonológica y el nivel de escritura silábico: Un estudio con niños preescolares. Revista de Filosofía y Psicología, 1(13), 81-100. 
Castles, A. \& Coltheart, M. (2004). Is there a causal link from phonological awareness to success in learning to read? Cognition, 91, 77-111.

Coolican, H. (1994). Métodos de Investigación y Estadística en Psicología. México: Manual Moderno.

Davey, A. \& Savla, J. (2010). Statistical power analysis with missing data: A structural equation modeling approach. Nueva York: Routledge.

Defior, S. (1990). Influencia de la codificación fonológica en el aprendizaje de la lectura. Universidad de Granada, Granada: Servicio de Publicaciones.

Defior, S. \& Serrano, F. (2011). La conciencia fonémica, aliada de la adquisición lenguaje escrito. Revista de Logopedia, Foniatría y Audiología, 31(1), 2-13.

Diuk, B., Borzone, A. M. \& Ledesma, R. (2010). Conocimiento de vocabulario, representaciones fonológicas y sensibilidad fonológica en niños pequeños de distinto sector social de procedencia. Summa Psicológica, 7(1), 33-50.

Domínguez, A. (1996). Evaluación de los efectos a largo plazo de la enseñanza de habilidades de análisis fonológico en el aprendizaje de la lectura y de la escritura. Infancia y Aprendizaje, 76, 69-81.

Fumagalli, J., Barreyro, J. P., Borzone, A. M. \& Jaichenco, V. (2014). Incidencia del tipo de unidad y la complejidad silábica en una tarea de conciencia fonológica. Estudios de Lingüistica Aplicada, 32(60), 35-55.

Gómez, L., Duarte, A., Merchán, V., Aguirre, D. \& Pineda, D. (2007). Conciencia fonológica y comportamiento verbal en niños con dificultades de aprendizaje. Universitas Psychologica, 6, 571-580.

González, R., López, S., Cuetos, F. \& Vilar, F. (2017). Efectos del entrenamiento en conciencia fonológica y velocidad de denominación sobre la lectura. Un estudio longitudinal. Estudios sobre educación, 32, 155-177.

Gutiérrez, R. (2016). La lectura dialógica como medio para la mejora de la comprensión lectora. Investigaciones Sobre Lectura, 5, 52-58.

Gutiérrez, R. (2018a). Habilidades favorecedoras del aprendizaje de la lectura en alumnos de 5 y 6 años. Revista Signos. Estudios de Lingüística, 51(96), 45-60. doi:10.4067/S0718-09342018000100045

Gutiérrez, R. (2018b). Estudio longitudinal sobre el fomento de las habilidades de escritura en el periodo de alfabetización inicial. Revista de Psicodidácica, 23(2), 137-143. doi: 10.1016/j.psicod.2017.09.002 
Gutiérrez, R. \& Díez, A. (2015). Aprendizaje de la escritura y habilidades de conciencia fonológica en las primeras edades. Bordón, 67(4), 43-59. doi: $\underline{10.13042 / \text { bordon.2015.67405 }}$

Gutiérrez, R. \& Díez, A. (2018). Conciencia fonológica y desarrollo evolutivo de la escritura en las primeras edades. Educación XX1, 21(1), 395-416. doi: 10.5944/educXX1.13256

Jasińska, K. \& Laura-Ann, P. (2017). Age of bilingual exposure is related to the contribution of phonological and semantic knowledge to successful reading development. Child Development, 89(1), 310-331.

Jiménez, J. E. \& Ortiz, M. R. (2000). Conciencia fonológica y aprendizaje de la lectura: Teoría, evaluación e intervención. Madrid: Síntesis.

Márquez, J. \& Osa, P. (2003). Evaluación de la conciencia fonológica en el inicio lector. Anuario Psicológico, 3, 357-370.

Martínez-Arias, M. R. (1995). Psicometría: Teoría de los Tests Psicológicos y Educativos. Madrid: Síntesis.

Meira, Â., Cadime, I. \& Leopoldina, F. (2019). The structure of phonological awareness in European Portuguese: A study of preschool children. The Journal of Educational Research, 112(3), 367-376. doi: 10.1080/00220671.2018.1530966

Pérez, M. \& González, M. (2004). Desarrollo del conocimiento fonológico, experiencia lectora y dificultad de la tarea. Revista de Logopedia, Foniatría y Audiología, 24(1), 2-15.

Ramos, J. L. \& Cuadrado, I. (2006). Prueba para la Evaluación del Conocimiento Fonológico. PECO. Madrid: EOS.

Rendón, S., García, E. \& Navarro, M. (2019). La lectura de palabras: La influencia del procesamiento fonológico y del método lectoescritor. Revista Fuentes, 21(1), 11-24. doi: 10.12795/revistafuentes.2019.v21.i1.01

Sellés, P., Martínez, T., Vidal-Abarca. E. \& Gilabert, R. (2008). BIL 3-6. Batería de Inicio a la lectura. Madrid: Instituto de Ciencias de la Educación.

Suárez-Coalla, P., García de Castro, M. \& Cuetos, F. (2013). Variables predictoras de la lectura y la escritura en castellano. Infancia y aprendizaje, 36(1), 77-89. doi:10.1174/021037013804826537

Thomson, J. \& Hogan, T. (2009). Future advances in the early detection of reading risk: Subgroups, dynamic relations, and advanced methodologies. Journal of Learning Disabilities, 43(4), 383-386. 
Treiman, R. \& Zukowski, A. (1991). Levels of phonological awareness. En S. A. Brady, \& D. P. Shankweiler (Eds.), Phonological processes in literacy. A tribute to Isabelle Y. Liberman (pp. 67-83). Hillsdale, NJ: Erlbaum.

Vicente-Yagüe, M. ${ }^{\mathrm{a}}$-I (2016). Artes y literatura en la LOMCE: Mecanismos para la comprensión de lecturas plurales. Investigaciones Sobre Lectura, 5, 59-69.

Ziegler, J. C. \& Goswami, U. (2005). Reading acquisition, developmental dyslexia, and skilled reading across languages: A psycholinguistic grain size theory. Psychological Bulletin, 131, 3-29. 